\title{
A Multiple-Technique Approach for Resolving the Surface Structure of Lithium and Manganese Rich Transition Metal Oxides.
}

\author{
Alpesh Khushalchand Shukla ${ }^{1}$, Quentin Ramasse ${ }^{2}$, Colin Ophus $^{3}$, Hugues Duncan ${ }^{4}$ and Guoying Chen ${ }^{1}$ \\ 1. Energy Storage and Distributed Resources Division, Lawrence Berkeley National Laboratory, \\ Berkeley, United States. \\ 2. SuperSTEM, Daresbury, United Kingdom. \\ 3. National Center of Electron Microscopy, Molecular Foundry, Lawrence Berkeley National \\ Laboratory, Berkeley United States. \\ 4. Kinestral Technologies, South San Francisco, United States
}

High-capacity lithium transition-metal oxides $\left(\mathrm{Li}_{1+\mathrm{x}} \mathrm{M}_{1-\mathrm{x}} \mathrm{O}_{2}\right.$, where $\mathrm{M}$ is usually a combination of transition metals such as $\mathrm{Mn}$, Co and $\mathrm{Ni}$ ) have been extensively studied recently due to their potential application in high-energy Li-ion batteries. Structural differences observed on the bulk and the surface, particularly the enrichment of $\mathrm{Ni}$ and/or Co and lower TM oxidation states on the surface, have been attributed to effects of cycling [1]. However, recent studies [2, 3] have shown that such transition metalrich surface layers are also observed in pristine materials. To ultimately achieve improved rate capability and stability of the batteries, it is necessary to fully understand the structure and composition of these surface layers. In this paper, we report the results from structural and spectroscopic analysis of these complex oxides using a variety of techniques that span a wide length-scale, including aberration corrected STEM, electron energy loss spectroscopy (EELS) and X-ray energy dispersive spectroscopy (XEDS).

$\mathrm{Li}_{1.2}\left(\mathrm{Ni}_{0.13} \mathrm{Mn}_{0.54} \mathrm{Co}_{0.13}\right) \mathrm{O}_{2}$ crystals with a plate-shaped morphology were prepared using a molten salt synthesis method and were studied using HAADF STEM imaging and EELS spectrum imaging in a Nion UltraSTEM electron microscope operating at $100 \mathrm{keV}$. XEDS experiments were conducted in an FEI Titan microscope equipped with a quad-silicon drift detector operating at $120 \mathrm{keV}$. HAADF STEM imaging using multiple zone axes revealed that the bulk of the primary particles consisted of variants of monoclinic phase, while the surface layer, which was typically $2 \mathrm{~nm}$ in thickness, had a spinel structure. An orientation relationship between the bulk and the spinel surface was found to be as follows:

$$
\left(\begin{array}{llll}
0 & 0 & 1
\end{array}\right)_{\mathrm{M}}\left\|\left(\begin{array}{lll}
1 & \overline{1} & 1
\end{array}\right)_{\mathrm{S}},\left[\begin{array}{lll}
0 & 1 & 0
\end{array}\right]_{\mathrm{M}}\right\|\left[\begin{array}{llll}
1 & 1 & 0
\end{array}\right]_{\mathrm{S}}
$$

$\mathrm{L}_{23}$ ratios obtained from EELS spectra across the surface revealed that the oxidation states of $\mathrm{Mn}$ and $\mathrm{Co}$, which was $4+$ and $3+$ in the bulk, decreased at the surface and the fine structure of $\mathrm{O}$ and Mn edge indicated the presence of spinel at the surface. EELS spectrum imaging also revealed higher concentration of $\mathrm{Co}$ and $\mathrm{Ni}$ on the surface. Analysis of Li K-edge showed a decreased concentration of $\mathrm{Li}$ at the surface, indicating the presence of antisite defects, which was also confirmed from HAADF STEM imaging. XEDS experiments confirmed the presence of Co and Ni segregation at the expense of Mn depletion along with depletion of oxygen at the surface, which explains the charge balance in the spinel surface in spite of lower oxidation states of Co and Mn. This result is consistent with earlier studies on oxygen-deficient spinels that have shown that spinels can easily accommodate oxygen vacancies [4].

Thus using a multiple-technique approach, we deduced that the surface of Li-rich layered oxides is a Co 
and Ni-rich, oxygen-deficient spinel with several anti-site defects.

\section{References:}

[1] B Xu et al, Energy \& Environmental Science 4 (2011) p. 2223.

[2] M Gu, et al, Nano Letters 12 (2012), p. 5186.

[3] H Dixit, et al, ACS Nano 8 (2014), p. 12710.

[4] A. Bergstein and P. Kleinert, Journal of Physics and Chemistry of Solids 26 (1965), p. 1181.

[5] The authors acknowledge support of the National Center of Electron Microscopy, Lawrence Berkeley Lab, which is supported by the U.S. Department of Energy under Contract \# DEAC02-05CH11231.

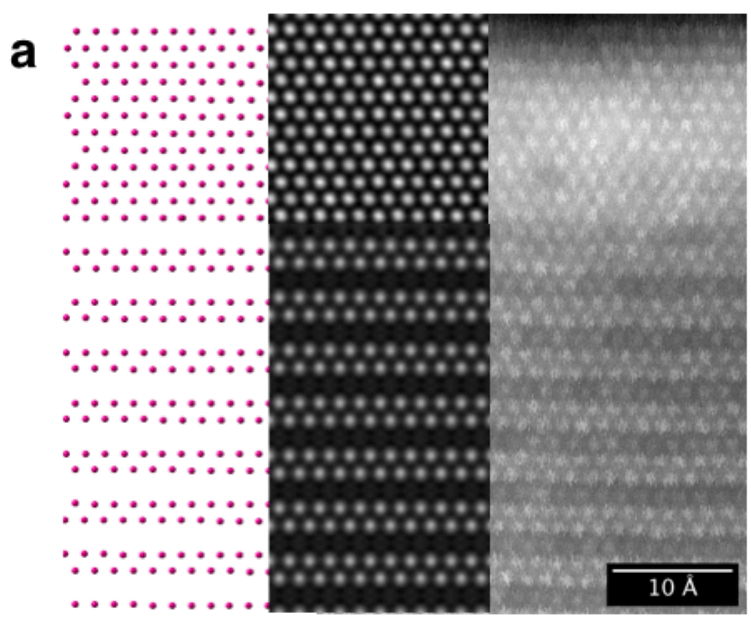

b
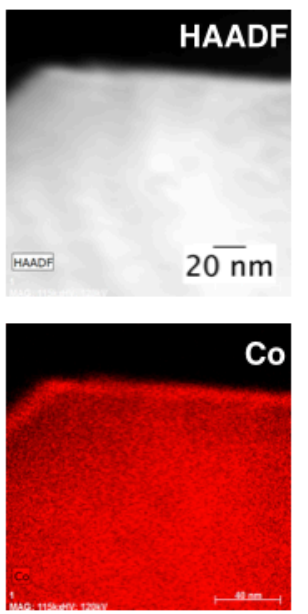
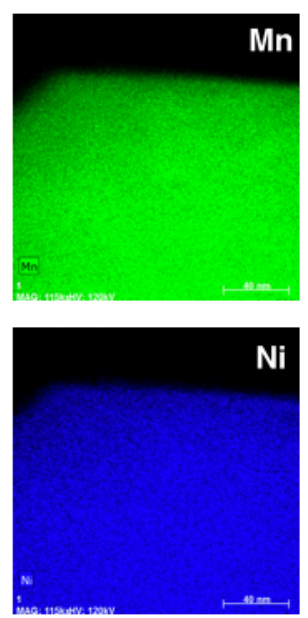

Figure 1. (a) Structural model, STEM simulation and experimental HAADF STEM image showing spinel structure on the surface and monoclinic structure in the bulk, in $[111]_{\mathrm{S}}$ and $[103]_{\mathrm{M}}$ zone axes, respectively. (b) XEDS maps showing enrichment of mostly Co and some Ni on the surface.
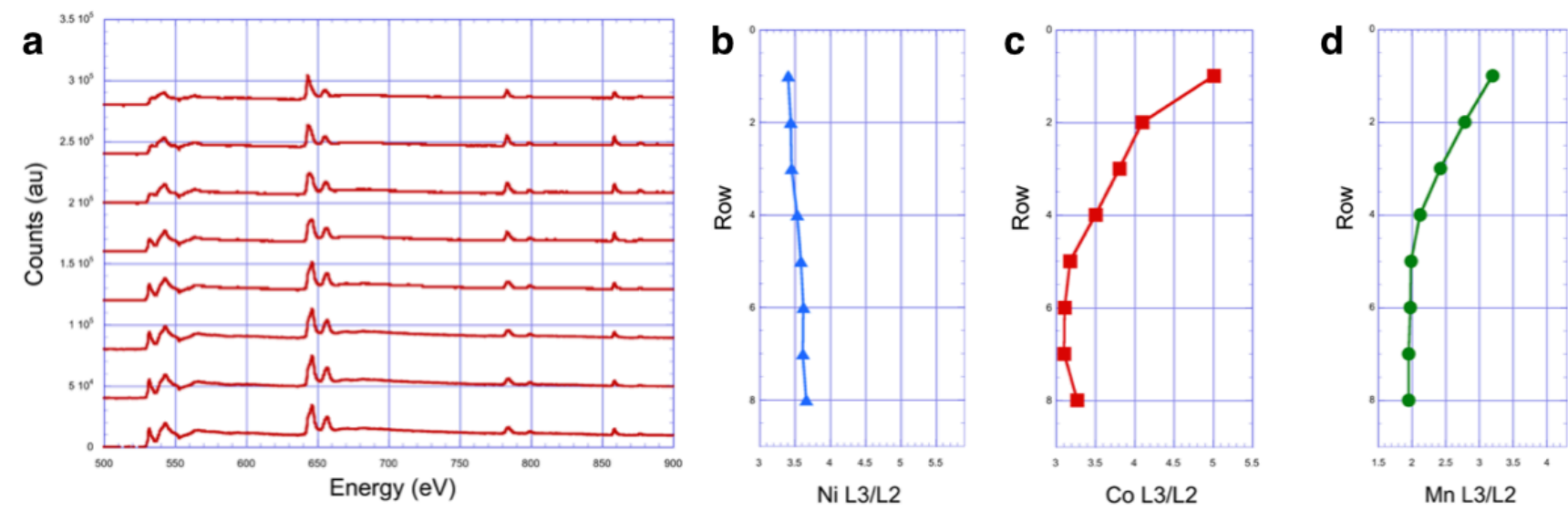

Figure 2. (a) Series of EEL spectra showing the surface (top) and the bulk (bottom) corresponding to the HAADF image in Figure 1a. (b), (c) and (d) $\mathrm{L}_{23}$ ratio calculated from (a) showing decreased oxidation states of $\mathrm{Mn}$ and $\mathrm{Co}$ at the surface. 